\title{
Hip bone mineral density, bone turnover and risk of fracture in patients on long-term suppressive L-thyroxine therapy for differentiated thyroid carcinoma
}

\author{
A Caroline Heijckmann ${ }^{1,2}$, Maya S P Huijberts ${ }^{1}$, Piet Geusens ${ }^{1}$, Jolanda de Vries ${ }^{3}$, Paul P C A Menheere ${ }^{4}$ \\ and Bruce H R Wolffenbuttel ${ }^{5}$ \\ ${ }^{1}$ Department of Internal Medicine, Division of Endocrinology, University Hospital Maastricht, Maastricht, The Netherlands, ${ }^{2}$ Department of Internal \\ Medicine, Hospital Bernhoven, Veghel/Oss, The Netherlands, ${ }^{3}$ Department of Psychology and Health, Tilburg University and St Elisabeth Hospital Tilburg, \\ Tilburg, The Netherlands ${ }^{4}$ Department of Clinical Chemistry, University Hospital Maastricht, Maastricht, The Netherlands and ${ }^{5}$ Department of \\ Endocrinology, University Medical Center Groningen, Groningen, The Netherlands
}

(Correspondence should be addressed to A C Heijckmann; Email: c.heijckmann@bernhoven.nl)

\begin{abstract}
Objective: Untreated hyperthyroidism and treatment with high doses of thyroid hormone are associated with osteoporosis. However, their effect on bone turnover, their contribution to bone mineral density (BMD) in the context of other clinical risk factors for osteoporosis and the prevalence of vertebral fractures is not well documented.

Design: Cross-sectional study.

Methods: We studied 59 patients receiving L-thyroxine suppressive therapy for differentiated thyroid carcinoma (DTC). BMD of the hip was measured by dual X-ray absorptiometry (DXA) and lateral DXA pictures of the lumbar and thoracic vertebrae were performed. Bone resorption was measured by C-telopeptides of type I collagen (ICTP) and bone formation by procollagen type I N-propeptide (PINP). Clinical risk factors for osteoporosis were evaluated using a questionnaire.

Results: Z-scores of BMD were similar as the NHANES (National Health and Nutrition Examination Survey) III reference group in women and men, also after long-term ( $>10$ years) suppression therapy. Patients in the lowest and highest quartile of BMD showed significant differences in the presence of clinical risk factors. ICTP levels were significantly higher than in age-matched controls, PINP levels were not different. We found four patients with a prevalent vertebral fracture.

Conclusions: We conclude that patients with well-differentiated thyroid carcinoma are not at increased risk of developing low bone mass nor have a higher prevalence of vertebral fracture at least when treated with relatively low doses of L-thyroxine.
\end{abstract}

European Journal of Endocrinology $15323-29$

\section{Introduction}

Patients with differentiated thyroid carcinoma (DTC) usually have a good prognosis and a near normal life expectancy. After initial ablative treatment with radioactive iodine these patients are treated with L-thyroxine (L-T4) in relatively high doses in order to suppress endogenous thyroid-stimulating hormone (TSH)-levels and to minimize potential TSH-mediated stimulation of tumor growth $(1,2)$. Adequate monitoring of TSH suppression has become possible since the development of second and third generation TSH assays $(3,4)$. The degree of TSH lowering required to obtain maximal suppression of tumor growth has been subject of discussion (5-8). Most recommendations suggest maintaining TSH levels lower than $0.1 \mathrm{mU} / \mathrm{l}(1)$, although in recent guidelines TSH levels between 0.1 and
$0.5 \mathrm{mU} / \mathrm{l}$ are recommended in case there is no residual disease after 1 year in low risk DTC patients (9). Longterm administration of supraphysiological doses of L-T4 may have adverse side-effects. In addition to physical discomfort, loss of weight, atrial fibrillation and cardiac dysfunction $(10,11)$, the possible negative effects on bone mineral density (BMD) and bone remodeling resulting in osteopenia and loss of bone strength are an important issue (12). Several mechanisms may be involved in the development of osteopenia secondary to suppressive therapy with L-T4 such as direct or indirect stimulation of osteoclast activity by this hormone. Recently also direct effects of TSH on bone remodeling have been described (13). TSH inhibits osteoclast formation and survival and also inhibits osteoblast differentiation, and is therefore a negative regulator of skeletal remodeling. Loss of bone mass is a uniform 
feature of overt hyperthyroidism; in several studies a bone loss of $10-20 \%$ has been reported (14-16). A history of overt hyperthyroidism is a risk factor for hip fracture later in life $(17,18)$, and is one of the causes of excess late mortality in previously hyperthyroid patients (19). Symptomatic bone disease is not a prominent feature of subclinical hyperthyroidism. However, mild (subclinical) hyperthyroidism in subjects with multinodular goiter was associated with decreased forearm bone density, while in postmenopausal women with hyperthyroidism who were treated with methimazole a higher bone density of the distal forearm was found compared with untreated women $(20,21)$. In addition the risk of osteoporotic fractures may be increased (22). Studies in patients with suppressive L-T4 therapy for DTC have generated conflicting results. Some authors (23-26) found no evidence of lower bone mass in this patient group, while others reported more or less deleterious effects on bone mass (27-29). In most of these studies however, numbers were small, patient selection was not well defined and characterization of patients was limited. The aim of our study was to evaluate the impact of long-term suppressive therapy with L-T4 on BMD in a large cohort of wellcharacterized patients with DTC, and in the subgroup of patients with suppressive therapy for more than 10 years. In addition, the prevalence of vertebral fractures was assessed using lateral spine radiographs and markers of bone formation and resorption were measured in serum.

\section{Subjects and methods}

\section{Patients}

Between January 2002 and July 2003 all 85 patients with DTC from the outpatient clinic of the University Hospital Maastricht (Maastricht, The Netherlands) were asked to take part in this cross-sectional study. Sixty patients agreed to participate. They all had undergone total thyroidectomy and radioactive iodine ablation and were taking a suppressive dose of L-T4 (goal of therapy: TSH $<0.05 \mathrm{mU} / \mathrm{l}$ ) for a median period of 5 years (range 1-52 years). In the last 6 months prior to entering this study, TSH-levels were below $0.05 \mathrm{mU} / \mathrm{l}$ in 32 patients and below $0.6 \mathrm{mU} / \mathrm{l}$ in the remaining subjects (mean TSH in the total group $0.06 \mathrm{mU} / \mathrm{l}$, range $<0.05-0.6 \mathrm{mU} / \mathrm{l})$. One patient who had a TSH-level of $6.1 \mathrm{mU} / \mathrm{l}$ was excluded from the study. Therefore complete data are available from 59 patients; written informed consent was obtained in all. To affirm that this patient group was under continuous suppressive therapy with L-T4 we checked serial serum TSH levels in a group of 32 patients with suppressive dose of L-T4 for $>5$ years resulting in a mean TSH of $0.06 \mathrm{mU} / \mathrm{l}$ (range $<0.05-0.35 \mathrm{mU} / \mathrm{l}$ ).

The general characteristics of the patients studied are summarized in Table 1 . Of the 40 women, 26 were premenopausal and 14 postmenopausal. All patients had histologically proven differentiated papillary or follicular thyroid cancer. FT4 levels were $21.9 \pm 3.3 \mathrm{pmol} / \mathrm{l}$ (reference parameter: $8-18 \mathrm{pmol} / \mathrm{l}$ ) and the mean dose of L-T4 therapy was $2.2 \pm 0.5 \mu \mathrm{g} / \mathrm{kg} /$ day. Ten patients used a vitamin D analogue and calcium supplementation because of postoperative hypoparathyroidism. No patient had taken estrogen replacement therapy or bisphosphonates. Calcium intake of all patients was assessed on the basis of a detailed dietary list. Risk factors for osteoporosis as well as daily activities and exercise were assessed by a validated questionnaire (30), in which sports, daily and work activities are scored with a minimum of zero and a maximum of eighteen. None of the 25 patients who declined to participate had impaired mobility or symptoms related to vertebral fractures. Mean age of this group was 49 years and there were seven postmenopausal women in this group.

\section{BMD and morphometric measurements}

BMD of the hip was measured by dual energy X-ray absorption (DXA, Hologic QDR 4500, Hologic Inc., Bedford, MA, USA, NHANES-III reference group). The

Table 1 Patients characteristics.

\begin{tabular}{lccc}
\hline & & & \\
& & Female & \\
\cline { 2 - 3 } & Premenopausal & Postmenopausal & Male \\
\hline Number & 26 & 14 & 19 \\
Age (years) & $40 \pm 7$ & $63 \pm 9$ & $52 \pm 12$ \\
BMI (kg/m ${ }^{2}$ ) & $24.4 \pm 3.2$ & $25.5 \pm 3.5$ & $28.8 \pm 3.2$ \\
Years of suppressive therapy (range) & $4(1-14)$ & $5.5(1-52)$ & $6(1-22)$ \\
Postop. hypoparathyroidism & 6 & 1 & 3 \\
Dose L-T4 ( $\mu$ g/kg/day) & $2.3 \pm 0.5$ & $1.9 \pm 0.4$ & $2.2 \pm 0.5$ \\
Papillary carcinoma & 13 & 8 & 12 \\
Follicular carcinoma & 10 & 4 & 6 \\
Mixed carcinoma & 3 & 2 & 1 \\
Serum $\mathrm{FT}_{4}$ (pmol/l) & $22.0 \pm 3.2$ & $22.7 \pm 3.8$ & $21.1 \pm 3.2$ \\
\hline
\end{tabular}

Data are expressed as numbers or means \pm S.D., numbers if appropriate or median (range). BMI, body mass index; postop., postoperative. 
hip was measured in the standard projection, and results are reported for femoral neck, trochanter, and total hip. No data on bone mineral density of the lumbar spine were obtained. Standard procedures supplied by the manufacturer for scanning and analysis were performed. Calibration with the manufacturer's spine phantom and quality control analysis was performed daily. The coefficient of variation for BMD measurements was $1.0 \%$. A lateral radiograph of the spine was made for evaluation of the presence of vertebral fractures. This was done by one classified and experienced person with morphometry. Anterior, middle and posterior height were recorded in all vertebrae from T4 to L4 (if possible). On the basis of these morphometric measurements prevalent vertebral deformity was defined as a reduction of height of $20 \%$ or more (McCloskey-Kanis method; 31).

\section{Laboratory assays}

All serum samples were frozen at $-20^{\circ} \mathrm{C}$ immediately after collection. All analyses were measured within one badge at the same time to reduce variability. Serum TSH was measured using the commercially available delayed enhanced Lantanide fluorescence immunometric assay (DELFIA-method, Perkin Elmer, Wallac, Turku, Finland), measured on an automatic immunoanalyser of the same manufacturer. Serum FT4 was measured using a commercially available solid phase fluoroimmunoassay (DELFIA-method, Perkin Elmer, Wallac, Turku, Finland); the normal reference range in our laboratory is $8-18 \mathrm{pmol} / \mathrm{l}$. As marker for bone formation procollagen serum level of type I amino-terminal propeptide (PINP) was measured and as marker for bone resorption serum level of carboxy-terminal cross-linked telopeptide of type I collagen (ICTP). PINP and ICTP were determined using commercial RIA kits (Orion Diagnostica Oy, Espoo, Finland). For PINP the interassay coefficient of variation was $3.2 \%$, the intraassay CV 2.5\% and the lowest detectable concentration was found to be $0.4 \mu \mathrm{g} / \mathrm{l}$. For ICTP the interassay CV was 3.5\%, the intraassay CV $2.3 \%$ and the lowest detectable concentration was found to be lower than $0.1 \mu \mathrm{g} / \mathrm{l}$. Study samples were compared with serum samples from age and sex-matched controls.
All gave informed consent. None of them were known to have thyroid diseases, and all had normal physical examination and normal results of routine lab measurements.

\section{Statistical analysis}

Results are reported as means \pm S.D. or median and (range) where appropriate. T- and Z-scores of the femoral neck and total hip (using NHANES-III) were calculated for the total group, for men, for pre- and postmenopausal women, and for a group of patients who received suppressive L-T4 therapy for 10 years or more. Comparison between groups was done using Student $t$-test with correction for multiple comparisons among groups if the data were normally distributed and by Kruskal-Wallis test if the data were not normally distributed. Pearson's correlations were used to calculate the relationship between BMD and duration of suppressive therapy. To assess the influence of confounding factors on the bone mineral density measurements, a multiple regression analysis (stepwise method) was performed with BMD of the total hip (Z-score) as a dependent variable and known risk factors for osteoporosis (sex, weight below $60 \mathrm{~kg}$, mother with hip fracture, fractures in previous medical history, postmenopausal state, and corticosteroid use), duration of suppressive therapy, body mass index (BMI), calcium intake, physical activity, L-T4/kg/day, serum FT4, and postoperative hypoparathyroidism as independent variables. Another multiple regression analysis (stepwise method) was done with BMD Z-scores as dependent variable and age, weight, L-T4/kg/day, serum FT4, number of risk factors and physical activity index as independent variables. Patients in the highest and lowest quartile of BMD T-scores were compared on presence of risk factors with a chi-square test. All analyses were done using SPSS (version 11.5). $P<0.05$ was considered statistically significant.

\section{Results}

\section{BMD and general characteristics}

Relative to the background population (NHANES reference group) bone mineral density was not decreased in

Table 2 Bone mineral density.

\begin{tabular}{lccr}
\hline & \multicolumn{3}{c}{ Female } \\
\cline { 2 - 3 } & Premenopausal & Postmenopausal & Male \\
\hline Number & 26 & 14 & 19 \\
L-T4 suppressive therapy* & 6 & 5 & 4 \\
Z score total hip & $0.44 \pm 0.87$ & $0.23 \pm 1.07$ & $0.37 \pm 0.84$ \\
Z score neck & $0.22 \pm 0.81$ & $0.12 \pm 0.95$ & $0.09 \pm 0.93$ \\
Z score trochanter & $0.61 \pm 1.1$ & $0.29 \pm 1.03$ & $0.49 \pm 0.91$ \\
\hline
\end{tabular}

Data are expressed as means \pm S.D. *for $\geq 10$ years. 
the three study groups (Table 2). Five patients (two men, three postmenopausal women) had a T-score $\leq-2.5$ in the femoral neck or trochanter and thus met the WHO-criteria for osteoporosis. Fifteen patients had suppressive L-T4 therapy for $\geqq 10$ years (median $14 \mathrm{yrs}$, range 10-52 yrs). Also in these patients no difference in BMD relative to age-matched controls (Z-score total hip: median 0.40 (range $-1.3-1.4$ ) was observed, and no correlation was found between the duration of suppressive therapy and BMD of the hip $(r=0.01, P=0.91)$. Moreover we compared the group of subjects with TSH levels below 0.05 with the patients who had a slightly higher TSH and these groups were similar with regards to T-score and Z-score of total hip. BMD of the hip was also not different in patients with or without postoperative hypoparathyroidism (Z-score 0.49 vs 0.34), although the Z-score of the femoral neck was slightly higher in patients with postoperative hypoparathyroidism $(0.70$ vs $0.05, P=0.029)$. Data regarding the presence of risk factors for osteoporosis and the daily calcium intake and physical activities have been summarized in Table 3. The activity index had a mean of nearly ten reflecting an average in showing activities in this patient group. The calcium intake is with a mean of $807 \mathrm{mg} /$ day moderate and insufficient for the postmenopausal group (in which $1000-1500 \mathrm{mg}$ is recommended to achieve zero calcium balance).

Multiple regression analysis, including age, weight, L-T4/kg per day, serum FT4, number of risk factors and physical activity, revealed that higher body weight was a significant contributor to BMD of the total hip as expressed as Z-score. There were 22 patients with in total 33 known clinical risk factors (postmenopausal state, hip fracture mother, vertebral fracture, fracture $>50$ year, body weight $<60 \mathrm{~kg}$, immobilization and corticosteroid use). Patients in the lowest and highest quartile of BMD (T-score of the total hip below -0.70 , mean -1.4 , and above 0.6 , mean 1.03) showed differences in the presence of clinical risk factors as earlier defined: in the lowest quartile,
11 of 15 patients had one or more risk factors, in the highest quartile, two of the 15 patients had any risk factor $(P=0.001)$ (Table 4$)$.

\section{Markers of bone formation and resorption}

Results of the measurement of markers for bone turnover are summarized in Table 5. Relative to an age matched control group, ICTP was increased by about $25 \%$ in all patient groups. PINP levels however, as marker of bone formation, did not significantly differ from the control group. Comparing patients with and without postoperative hypoparathyroidism, markers of bone formation $(-44 \%, P<0.05)$ and resorption $(-7 \%)$ were decreased in the hypoparathyroidism group and this resulted in a slightly higher BMD mainly in the femoral neck.

\section{Vertebral fractures}

Fifty-four lateral DXA pictures of the lumbar and thoracic vertebrae were of adequate quality for the identification of vertebral fractures. Four patients were found to have pre-existing vertebral fracture, i.e. $7 . \%$ of patients; two men, one pre- and one postmenopausal woman. These patients did not report earlier fractures or pain episodes. All these patients had a BMD of the hip within the age-matched reference range (Z-score $0.53 \pm 0.91)$.

\section{Discussion}

This study demonstrates that long-term suppressive L-T4 therapy in patients with DTC does not result in reduced bone mineral density of the hip, neither in pre- and postmenopausal women nor in men. BMD, expressed as Z-score, was not correlated with duration of L-T4 therapy and even in patients with suppressive therapy $>10$ years no significant decrease in BMD was found. The goal of suppressive L-T4 therapy is to

Table 3 Risk factors and questionnaires.

\begin{tabular}{lccc}
\hline & \multicolumn{3}{c}{ Female } \\
\cline { 2 - 3 } & Premenopausal & Postmenopausal & Male \\
\hline Number & 26 & 14 & 19 \\
Years since menopause & & $12 \pm 10$ & 1 \\
Fracture $>50$ years & 0 & 1 & 2 \\
Vertebral fracture & 1 & 4 & 1 \\
Hip fracture mother & 0 & 5 & 0 \\
Low body weight (<60 kg) & 5 & 0 & 0 \\
Immobilisation & 0 & 0 & $822 \pm 326$ \\
Corticosteroids in last 10 years & 0 & $782 \pm 290$ & $9.9 \pm 3.6$ \\
Calcium intake (mg/day) & $810 \pm 357$ & $8.6 \pm 4.1$ & \\
Physical activity (elders) & $10.6 \pm 4.0$ & & 0 \\
\hline
\end{tabular}

Data are expressed as numbers or means \pm S.D. 
Table 4 Number of patients with clinical risk factors in all 4 quartiles of BMD (T-scores).

\begin{tabular}{lcc}
\hline & $\begin{array}{c}\text { No. of patients } \\
\text { in quartile }\end{array}$ & $\begin{array}{c}\text { No. of patients with } \\
\geq \mathbf{1} \text { clinical risk factor }\end{array}$ \\
\hline T-score $\leq-0.7$ & 15 & 11 \\
$-0.7<\mathrm{T}$-score $\leq 0$ & 15 & 7 \\
$0<\mathrm{T}$-score $\leq 0.5$ & 14 & 2 \\
T-score $>0.5$ & 15 & 2 \\
\hline
\end{tabular}

maintain TSH levels $<0.05 \mathrm{mU} / \mathrm{l}$ with a high normal FT4. In our study the dose needed to achieve this level of suppression was $2.2 \mu \mathrm{g} / \mathrm{kg}$ of body weight per day, which resulted in FT4 levels of $20 \%$ above the upper limit of normal. In all studies on this subject in which a significant bone loss was observed patients used a 30-50\% higher dose of L-T4 compared with our patients $(27-29,32,33)$. In a cross-sectional study Kung et al. found significantly lower BMD in the lumbar spine, femoral neck and trochanter $(-18 \%,-12 \%$ and $-13 \%)$ in a group of 34 postmenopausal women, and their L-T4 dose was $3.3 \mu \mathrm{g} / \mathrm{kg}$ of body weight per day (28). The same group followed a small group of 15 postmenopausal women $(3.0 \mu \mathrm{g}$ L-T4/kg of body weight per day) and found a bone loss of $6.7 \%$ in the total hip after 2 years (32), which could be prevented by calcium supplementation (1000 mg/day). Diamond et al. demonstrated a negative effect on BMD in both pre- and postmenopausal women $(n=24)$ (27) with a mean L-T4 dose of $2.8 \mu \mathrm{g} / \mathrm{kg} / \mathrm{day}$ and found a significant correlation between the total cumulative dose of L-T4 and BMD, suggesting that overzealous L-T4 therapy may lead to bone loss. Studies in which doses lower than $2.5 \mu \mathrm{g} / \mathrm{kg}$ body weight per day were used, including the more recent study of Rosen et al. $(24,26,34)$ did not observe any negative effects on BMD. Before the introduction of second and third generation TSH assays in the late eighties, it was more difficult to monitor suppressive L-T4 therapy, because the detection limit was within the normal range. Therefore dosing of L-T4 could not be titrated optimally and was probably unnecessarily high. These high L-T4 doses have been shown to affect BMD $(27-29,32)$. The presently available TSH assays are more sensitive and thereby allow better dose titration, thereby preventing unwanted loss of BMD. The presence of postoperative parathyroid dysfunction could also contribute to the low incidence of osteoporosis in our population. Parathyroid hormone (PTH) is generally thought to have a net catabolic effect on bone, including a more pronounced stimulatory effect on osteoclast than on osteoblast activity (35). Abugassa et al. found a higher bone mass in the proximal femur, lumbar spine and distal radius in a group of 13 patients treated with calcium and/or dihydrotachysterol because of postoperative hypoparathyroidism after thyroidectomy for DTC, compared with patients with normal parathyroid function after the same surgical procedure (36). We also found in the ten subjects with postsurgical hypoparathyroidism a significantly higher BMD of the femoral neck. However the total hip measurements showed no difference between these two groups, and mean Z-scores in the group without hypoparathyroidism were not lower than the reference population. A total of five patients met the WHO criteria for osteoporosis in the measurements of total hip, trochanter or femoral neck BMD. Our risk factor analysis indicated that this group could be identified with the known clinical risk factors such as postmenopausal state, mother with hip fracture, fracture above 50 years, immobilisation, corticosteroid use and low body weight. Although no long-term effect on BMD was found, a significant increase of bone resorption as measured by serum levels of ICTP was observed. The marker of bone formation PINP was not altered. Other studies in DTC patients with suppressive L-T4 therapy reported an increase in markers of both bone formation and resorption. Kung et al. reported increases in hydroxyproline in the urine of $130 \%$ and osteocalcin levels of $150 \%$ (28) suggesting increased bone turnover. It is not clear why this change in balance between bone formation and bone resorption in our patients did not lead to bone loss in the longterm. Perhaps the increase in bone resorption is not substantial enough to affect BMD. One might also speculate that the compliance with L-T4 therapy might be lower in between hospital visits $(37,38)$. Also a catch-up effect might have occurred in the periods of thyroid hormone withdrawal and subsequent hypothyroidism for diagnosis or treatment of recurrent or metastatic disease. Vertebral fracture is a hallmark of osteoporosis. Only one of the previous studies in patients with DTC has evaluated prevalent vertebral fractures in a very small group $(n=12)$ and reported no significant difference in the incidence of spinal deformity between TSH-suppressed

Table 5 Bone markers total group (in serum in $\mu \mathrm{g} / \mathrm{l}$ ).

\begin{tabular}{ccccccccc}
\hline & \multicolumn{2}{c}{ Female } & & \multicolumn{2}{c}{ Male } \\
\cline { 2 - 4 } \cline { 7 - 8 } & Premenopausal $n=24$ & Controls $n=24$ & Postmenopausal $n=10$ & Controls $n=10$ & Patients $n=19$ & Controls $n=19$ \\
\hline ICTP & $3.8 \pm 1.3^{*}$ & $3.0 \pm 7$ & $4.4 \pm 1.0^{*}$ & $3.3 \pm 1.1$ & & $4.1 \pm 0.9^{*}$ & $3.5 \pm 0.7$ \\
PINP & $31.0 \pm 17.3$ & $28.4 \pm 10.1$ & $39.7 \pm 21.1$ & $42.8 \pm 20.9$ & & $36.8 \pm 15.5$ & $37.2 \pm 11.3$ \\
\hline
\end{tabular}

${ }^{\star} P<0.05$ vs age matched controls. ICTP, carboxy-terminal cross-linked telopeptide of type I collagen; PINP, type I amino-terminal propeptide. 
patients and nonsuppressed patients (39). We found four patients in this group $(7 \%)$ with one or more vertebral fractures defined as a reduction of $20 \%$ or more of the anterior, middle or posterior height. In a very large cross-sectional population based study on the prevalence of vertebral deformity in European men and women (EVOS) in a group of 15570 males and females aged 50-79 years, a prevalent vertebral deformity was observed in 12\% (range 6-21\%) (40). We report $7 \%$ in a group with lower mean age; these patients did not have any clinical sign of vertebral fracture nor did they have a lower BMD of the hip. Although the group is small we have no indication that the prevalence of vertebral fracture is higher than in a European reference population.

We conclude that L-T4 therapy in doses which suppress TSH just below the detection limit of a second generation TSH assay, did not result in significant bone loss in our studied population and probably also not in increased fracture risk. We suggest that specific screening for osteoporosis in this patient group is therefore not recommended, other than on the basis of the known clinical risk factors.

\section{References}

1 Pujol P, Daures JP, Nsakala N, Baldet L, Bringer J \& Jaffiol C. Degree of thyrotropin suppression as a prognostic determinant in differentiated thyroid cancer. Journal of Clinical Endocrinology and Metabolism 199681 4318-4323.

2 Cooper DS, Specker B, Ho M, Sperling M, Ladenson PW, Ross DS, Ain KB, Bigos ST, Brierley JD, Haugen BR, Klein I, Robbins J, Sherman SI, Taylor T \& Maxon HR III. Thyrotropin suppression and disease progression in patients with differentiated thyroid cancer: results from the National Thyroid Cancer Treatment Cooperative Registry. Thyroid $19988737-744$.

3 Demers LM \& Spencer CA. Laboratory medicine practice guidelines: laboratory support for the diagnosis and monitoring of thyroid disease. Clinical Endocrinology 200358 138-140.

4 Ross DS. Serum thyroid-stimulating hormone measurement for assessment of thyroid function and disease. Endocrinology and Metabolism Clinics of North America 200130 (vii) 245-264.

5 Wartofsky L. Use of sensitive TSH assay to determine optimal thyroid hormone therapy and avoid osteoporosis. Annual Review of Medicine 199142 341-345.

6 Lamberg BA, Helenius T \& Liewendahl K. Assessment of thyroxine suppression in thyroid carcinoma patients with a sensitive immunoradiometric TSH assay. Clinical Endocrinology 198625 259-263.

7 Burmeister LA, Goumaz MO, Mariash CN \& Oppenheimer JH. Levothyroxine dose requirements for thyrotropin suppression in the treatment of differentiated thyroid cancer. Journal of Clinical Endocrinology and Metabolism 199275 344-350.

8 Spencer CA, Lai-Rosenfeld AO, Guttler RB, LoPresti J, Marcus AO, Nimalasuriya A, Eigen A, Doss RC, Green BJ \& Nicoloff JT. Thyrotropin secretion in thyrotoxic and thyroxine-treated patients: assessment by a sensitive immunoenzymometric assay. Journal of Clinical Endocrinology and Metabolism 198663 349-355.

9 Schlumberger M, Berg G, Cohen O, Duntas L, Jamar F, Jarzab B, Limbert E, Lind P, Pacini F, Reiners C, Sanchez FF, Toft A \& Wiersinga WM. Follow-up of low-risk patients with differentiated thyroid carcinoma: a European perspective. European Journal of Endocrinology 2004150 105-112.
10 Biondi B, Fazio S, Carella C, Amato G, Cittadini A, Lupoli G, Sacca L, Bellastella A \& Lombardi G. Cardiac effects of long term thyrotropin-suppressive therapy with levothyroxine. Journal of Clinical Endocrinology and Metabolism 199377 334-338.

11 Sawin CT, Geller A, Wolf PA, Belanger AJ, Baker E, Bacharach P, Wilson PW, Benjamin EJ \& D'Agostino RB. Low serum thyrotropin concentrations as a risk factor for atrial fibrillation in older persons. New England Journal of Medicine 1994331 1249-1252.

12 Greenspan SL \& Greenspan FS. The effect of thyroid hormone on skeletal integrity. Annals of Internal Medicine 1999130 750-758.

13 Abe E, Marians RC, Yu W, Wu XB, Ando T, Li Y, Iqbal J, Eldeiry L, Rajendren G, Blair HC, Davies TF \& Zaidi M. TSH is a negative regulator of skeletal remodeling. Cell 2003115 151-162.

14 Linde J \& Friis T. Osteoporosis in hyperthyroidism estimated by photon absorptiometry. Acta Endocrinologica $1979 \quad 91$ 437-448.

15 Toh SH, Claunch BC \& Brown PH. Effect of hyperthyroidism and its treatment on bone mineral content. Archives of Internal Medicine $1985145883-886$.

16 Diamond T, Vine J, Smart R \& Butler P. Thyrotoxic bone disease in women: a potentially reversible disorder. Annals of Internal Medicine 1994120 8-11.

17 Cummings SR, Nevitt MC, Browner WS, Stone K, Fox KM, Ensrud KE, Cauley J, Black D \& Vogt TM. Risk factors for hip fracture in white women. Study of Osteoporotic Fractures Research Group. New England Journal of Medicine 1995332 $767-773$.

18 Wejda B, Hintze G, Katschinski B, Olbricht T \& Benker G. Hip fractures and the thyroid: a case-control study. Journal of Internal Medicine $1995237241-247$.

19 Franklyn JA, Maisonneuve P, Sheppard MC, Betteridge J \& Boyle P. Mortality after the treatment of hyperthyroidism with radioactive iodine. New England Journal of Medicine 1998338 712-718.

20 Mudde AH, Reijnders FJ \& Kruseman AC. Peripheral bone density in women with untreated multinodular goitre. Clinical Endocrinology $19923735-39$.

21 Mudde AH, Houben AJ \& Nieuwenhuijzen Kruseman AC. Bone metabolism during anti-thyroid drug treatment of endogenous subclinical hyperthyroidism. Clinical Endocrinology $1994 \mathbf{4 1}$ $421-424$.

22 Bauer DC, Ettinger B, Nevitt MC \& Stone KL. Risk for fracture in women with low serum levels of thyroid-stimulating hormone. Annals of Internal Medicine 2001134 561-568.

23 Franklyn JA, Betteridge J, Daykin J, Holder R, Oates GD, Parle JV, Lilley J, Heath DA \& Sheppard MC. Long-term thyroxine treatment and bone mineral density. Lancet 1992340 9-13.

24 Florkowski CM, Brownlie BE, Elliot JR, Ayling EM \& Turner JG. Bone mineral density in patients receiving suppressive doses of thyroxine for thyroid carcinoma. New Zealand Medical Journal $1993106443-444$.

25 Hawkins F, Rigopoulou D, Papapietro K \& Lopez MB. Spinal bone mass after long-term treatment with L-thyroxine in postmenopausal women with thyroid cancer and chronic lymphocytic thyroiditis. Calcified Tissue International 199454 16-19.

26 Rosen HN, Moses AC, Garber J, Ross DS, Lee SL, Ferguson L, Chen V, Lee K \& Greenspan SL. Randomized trial of pamidronate in patients with thyroid cancer: bone density is not reduced by suppressive doses of thyroxine, but is increased by cyclic intravenous pamidronate. Journal of Clinical Endocrinology and Metabolism $1998832324-2330$.

27 Diamond T, Nery L \& Hales I. A therapeutic dilemma: suppressive doses of thyroxine significantly reduce bone mineral measurements in both premenopausal and postmenopausal women with thyroid carcinoma. Journal of Clinical Endocrinology and Metabolism 199172 1184-1188.

28 Kung AW, Lorentz T \& Tam SC. Thyroxine suppressive therapy decreases bone mineral density in post-menopausal women. Clinical Endocrinology 199339 535-540.

29 Pioli G, Pedrazzoni M, Palummeri E, Sianesi M, Del FR, Vescovi PP, Prisco M, Ulietti V, Costi D \& Passeri M. Longitudinal study of 
bone loss after thyroidectomy and suppressive thyroxine therapy in premenopausal women. Acta Endocrinologica $1992 \mathbf{1 2 6}$ $238-242$.

30 Elders PJ, Netelenbos JC, Lips P, Khoe E, Van Ginkel FC, Hulshof KF \& Van der Stelt PF. Perimenopausal bone mass and risk factors. Bone and Mineral 19897 289-299.

31 McCloskey EV, Spector TD, Eyres KS, Fern ED, O'Rourke N, Vasikaran S \& Kanis JA. The assessment of vertebral deformity: a method for use in population studies and clinical trials. Osteoporos International $19933138-147$.

32 Kung AW \& Yeung SS. Prevention of bone loss induced by thyroxine suppressive therapy in postmenopausal women: the effect of calcium and calcitonin. Journal of Clinical Endocrinology and Metabolism 199681 1232-1236.

33 Muller CG, Bayley TA, Harrison JE \& Tsang R. Possible limited bone loss with suppressive thyroxine therapy is unlikely to have clinical relevance. Thyroid 19955 81-87.

34 Gorres G, Kaim A, Otte A, Gotze M \& Muller-Brand J. Bone mineral density in patients receiving suppressive doses of thyroxine for differentiated thyroid carcinoma. European Journal of Nuclear Medicine 199623 690-692.

35 Rodan GA \& Martin TJ. Role of osteoblasts in hormonal control of bone resorption-a hypothesis. Calcified Tissue International 1981 33 349-351.
36 Abugassa S, Nordenstrom J, Eriksson S \& Sjoden G. Bone mineral density in patients with chronic hypoparathyroidism. Journal of Clinical Endocrinology and Metabolism 199376 1617-1621.

37 Hughes CM. Medication non-adherence in the elderly: how big is the problem? Drugs and Aging 200421 793-811.

38 Barat I, Andreasen F \& Damsgaard EM. Drug therapy in the elderly: what doctors believe and patients actually do. British Journal of Clinical Pharmacology 200151 615-622.

39 Fujiyama K, Kiriyama T, Ito M, Kimura H, Ashizawa K, Tsuruta M, Nagayama Y, Villadolid MC, Yokoyama N \& Nagataki S. Suppressive doses of thyroxine do not accelerate age-related bone loss in late postmenopausal women. Thyroid 19955 13-17.

40 O'Neill TW, Felsenberg D, Varlow J, Cooper C, Kanis JA \& Silman AJ. The prevalence of vertebral deformity in european men and women: the European Vertebral Osteoporosis Study. Journal of Bone and Mineral Research 199611 1010-1018.

Received 31 December 2004

Accepted 22 March 2005 